\title{
LINEARLY ORDERABLE SPACES ${ }^{1}$
}

\section{L. LYNN}

A linearly ordered space $(X, T,<)$ is a set $X$ on which a linear ordering $<$ has been defined such that the interval topology generated by < agrees with $T$. (A subbasic set for the interval topology induced by $<$ is everything less than or greater than a given point.)

A space $(X, T)$ is linearly orderable if there is a linear ordering < on $X$ whose associated interval topology agrees with $T$.

The subspace $\{-1\} \cup\left\{n^{-1}: n=1,2, \cdots\right\}$ of the real line $R$ is not linearly ordered with respect to the usual ordering of $R$ because $\{-1\}$ is open in the relative topology but not in the interval topology. However, this subspace is homeomorphic to the linearly ordered space $\left\{n^{-1}: n=1,2, \cdots\right\}$, so it is linearly orderable.

The subspace $(0,1) \cup\{2\}$ of $R$ is, however, not even linearly orderable.

To the best of my knowledge there are no known sufficient conditions for a disconnected space to be linearly orderable. In the connected case the only affirmative results are the well-known characterizations of the arc (cf., e.g. [2, p. 168]) and a result of Eilenberg [1]. The purpose of this note is to prove the following:

THEOREM. Every zero-dimensional, separable metrizable space is linearly orderable.

Proof. Without loss of generality we may assume our space $(X, T)$ is a subset of the Cantor set $C$ in the unit interval $I$ [3, p. 173]. We will construct a homeomorphism $f$ of $X$ into $C$ which maps a countable dense subset of $X$ into a carefully selected subset of those points of $C$ which are end points of intervals of $R \sim C$. We will then show that in $f[X]$ :

(a) each point is the $T$-limit of a monotone increasing sequence of points of $f[X]$, or has an immediate predecessor, or is the minimum of $f[X]$, and

(b) each point is the $T$-limit of a monotone decreasing sequence of points of $f[X]$, or has an immediate successor, or is the maximum of $f[X]$.

The $T$ and interval topologies coincide precisely when (a) and (b) hold.

Presented to the Society April 14, 1961, under the title Linearly orderable subspaces of the real line; received by the editors May 15, 1961.

1 This research was supported by the Office of Naval Research and is part of the author's doctoral dissertation. 
Select a point $a_{0}$ in $X$.

Let $I_{0}$ be that closed end third of $I$ containing $a_{0}$ and $I_{2}$ be the other. If $X I_{2} \neq \varnothing$, select a point $a_{1}$ in $X I_{2}$.

Let $I_{00}$ be that closed end third of $I_{0}$ containing $a_{0}$ and $I_{02}$ be the other. If $X I_{02} \neq \varnothing$, select a point $a_{1 / 3}$ in $X I_{02}$. Similarly let $I_{22}$ be that closed end third of $I_{2}$ containing $a_{1}$ and $I_{20}$ be the other. If $X I_{20} \neq \varnothing$, select a point $a_{2 / 3}$ in $X I_{20}$.

Proceeding inductively, for each integer $n>1$, define intervals $I_{p_{1} \cdots p_{n}}\left(p_{i}=0\right.$ or 2 for $\left.i=1,2, \cdots, n\right)$ and, if $X I_{p_{1} \cdots p_{n}} \neq \varnothing$, a selected point in $X I_{p_{1}} \cdots p_{n}$ subject to the following restrictions:

$I_{p_{1}} \ldots p_{n}$ is a closed end third of $I_{p_{1}} \ldots p_{n-1}$.

The selected point in $X I_{p_{1} \cdots p_{n}}$ is the selected point in $X I_{p_{1} \cdots p_{n-1}}$ if $p_{n}=p_{n-1}$.

The selected point in $X I_{p_{1}} \ldots p_{n}$ has index $u \cdot 3^{-n+1}+\sum_{i=1}^{n-1} p_{i} \cdot 3^{-i}$, where $u=0$ if $p_{n}=0$, and $u=1$ otherwise.

Note that for each $a \in X$, there is a unique sequence of intervals $\left\{I_{p_{1}} \ldots p_{n}\right\}$ such that $\{a\}=\bigcap_{n=1}^{\infty} I_{p_{1} \cdots p_{n}}$. Thus, we may define a mapping $f: X \rightarrow C$ by letting $f(a)=\sum_{i=1}^{\infty} p_{i} \cdot 3^{-i}$.

If $a$ and $b$ are distinct points of $X$, there is an integer $j$ such that $p_{j} \neq q_{j}$. Thus, $\sum_{i=1}^{\infty} p_{i} \cdot 3^{-i} \neq \sum_{i=1}^{\infty} q_{i} \cdot 3^{-i}$. So, $f$ is a one-one mapping.

Moreover, $f$ is continuous. For, if $\left\{b_{m}\right\}$ is a sequence of points of $X$ converging to $a \in \bigcap_{n=1}^{\infty} I_{p_{1} \ldots p_{n}}$, then there is an integer $N_{n}$ such that both $b_{m}$ and $a$ are in $I_{p_{1}} \ldots p_{n}$ if $m \geq N_{n}$, whence both $f\left(b_{m}\right)$ and $f(a)$ are in $\left[\sum_{i=1}^{n} p_{i} \cdot 3^{-i}, 3^{-n}+\sum_{i=1}^{n} p_{i} \cdot 3^{-i}\right]$. A similar argument shows that $f^{-1}$ is also continuous.

We will show next that if a point of $f[X]$ which is an end point of an interval of $R \sim C$ is not a $T$-limit point from above (below), then it is not a limit point from above (below) in the interval topology. The converse is clear. It is easily seen from the nature of the mapping $f$ that each point of $f[X]$ which is not an end point of an interval of $R \sim C$ is a two-sided $T$-limit point of $f[X]$. So, this will conclude the proof.

Let $x=\sum_{i=1}^{r} p_{i} \cdot 3^{-i} \in f[X]$, where $p_{r} \neq 0$. Thus $p_{r}=1$ or 2 , and $p_{i}=0$ or 2 for $i \neq r$. Clearly $x$ is a $T$-limit point from at most one side. Thus, by symmetry, it suffices to prove our assertion in case $p_{r}=2$ and $x$ is not a $T$-limit point from above. The details of the following argument may be filled in easily.

Let $y=\inf \{z \in f[X]: x<z\}$. Suppose first that there is a largest integer $n_{0}>r$ such that $\alpha=x+3^{-n_{0}} \in f[X]$. If there is also a smallest $s_{0}$ such that $\beta=x+\sum_{i=n_{0}+1}^{s_{0}} 2 \cdot 3^{-i} \in f[X]$, then $y=\beta$, while if there is no such $s_{0}$, then $y=\alpha$. If there is no such $n_{0}$ and there is a smallest $s_{0}$ such that $\gamma=x+\sum_{i=r+1}^{s_{0}} 2 \cdot 3^{-i} \in f[X]$, then $y=\gamma$, while if there is 
no such $s_{0}$ then $y=3^{-r+1}+\sum_{i=1}^{r-1} p_{i} \cdot 3^{-i} \in f[X]$. Thus $y$ is the immediate successor of $x$.

Let $w=\sup \{z \in f[X]: z<x\}$, let $u_{j}=\sum_{i=1}^{r-j} p_{i} \cdot 3^{-i}$ for $j=1,2, \cdots$, $r-1$, let $u_{r}=0$, and $u_{k}$ be that $u_{j}(j=1,2, \cdots, r)$ with least index in $f[X]$. If there is a least $n_{0} \geq r-k+1$ such that $\delta=u_{k}+3^{-n_{0}} \in f[X]$, then $w=\delta$, while if there is no such $n_{0}$, then $w=u_{k}$. Thus $w$ is the immediate predecessor of $x$.

A similar procedure is followed in case $x=0$ or 1 .

In a future note the case of arbitrary subspaces of $R$ will be discussed. (See [4].)

\section{REFERENCES}

1. S. Eilenberg, Ordered topological spaces, Amer. J. Math. 63 (1941), 39-45.

2. D. W. Hall and G. L. Spencer, Elementary topology, Wiley, New York, 1955.

3. C. Kuratowski, Topologie, Vol. I, 4th ed., Warsaw, 1958.

4. I. L. Lynn, Linearly orderable subspaces of the real line, Notices Amer. Math. Soc. 8 (1961), 161.

Wayne State University and

PURDUe University 